\title{
Characteristics of Frozen-Thawed Spermatozoa Cryopreserved with Different Concentrations of Glycerol in Captive Japanese Black Bears (Ursus thibetanus japonicus)
}

\author{
Tsukasa OKANO ${ }^{1)}$, Sachiko NAKAMURA ${ }^{1)}$, Takeshi KOMATSU'), Tetsuma MURASE ${ }^{1,3)}$, Kiyoshi MIYAZAWA ${ }^{1,3)}$, \\ Makoto ASANO ${ }^{1,4)}$ and Toshio TSUBOTA ${ }^{1,4)^{*}}$ \\ ${ }^{1)}$ United Graduate School of Veterinary Sciences, Gifu University, Gifu 501-1193, ${ }^{2)}$ The Institute of Japanese Black Bear in Ani, Akita \\ 018-4731, ${ }^{3)}$ Laboratory of Theriogenology, Faculty of Applied Biological Sciences, Gifu University, Gifu 501-1193 ${ }^{4)}$ Laboratory of Zoo \\ and Wildlife Medicine, Faculty of Applied Biological Sciences, Gifu University, Gifu 501-1193, Japan
}

(Received 16 March 2006/Accepted 1 June 2006)

ABSTRACT. Seven mature Japanese black bears were used as semen donors, and a total of 7 semen samples collected from the animals by the electroejaculation method were cryopreserved in liquid nitrogen. Egg yolk-TRIS-citrate-glucose extender was used, and the effects of different final concentrations of glycerol, at $4-12 \%(\mathrm{v} / \mathrm{v})$, on frozen-thawed spermatozoa were examined. No significant difference was observed in percent motility or percent abnormal morphology of frozen-thawed spermatozoa among the different glycerol concentrations. Percent viability and percent intact acrosomes of spermatozoa cryopreserved with 4 and $6 \%$ glycerol were significantly higher than those with 10 and $12 \%$ glycerol. These results suggest that a suitable glycerol concentration for freezing Japanese black bear semen within the range tested would be $4-6 \%$.

KEY WORDS: glycerol, Japanese black bear, semen cryopreservation.

The Japanese black bear (Ursus thibetanus japonicus) is an endangered species in some areas of Japan [5]. Semen cryopreservation is one of the most important techniques for species conservation as a genetic resource. There are reports of semen cryopreservation in ursids, including giant pandas (Ailuropoda melanoleuca) [13, 14, 18], Hokkaido brown bears (Ursus arctos yesoensis) [7], and Japanese black bears $[11,12]$. However, although efforts to establish an optimal technique are important, no comparative studies of different glycerol concentrations of diluents for bear semen cryopreservation have been reported. Glycerol has been widely used as a cryoprotectant in semen cryopreservation in domestic and non-domestic animals [19], although optimal glycerol concentrations vary among species [1]. Glycerol, a cryoprotective agent, exerts toxic effects on spermatozoa, such as plasma membrane rupture and acrosomal damage $[6,10]$.

The objective of the present study was to examine the effect of different glycerol concentrations of diluents for semen cryopreservation in Japanese black bears.

Seven mature male Japanese black bears over 4 years of age at Ani Mataginosato Bear Park, Akita, Japan (N 40, $\mathrm{E}$ $140.4^{\circ}$ ), were used as semen donors. They had been kept with 6 females and approximately 30 other males and had been allowed to mate freely with the females. Semen was collected once from each animal by electroejaculation during the mating season in June and July 2005. Electroejaculation was conducted according to the method of Kojima et al. [9] and our previous study [12] with some modifications.

\footnotetext{
* Correspondence to: Tsubota, T., United Graduate School of Veterinary Sciences, Gifu University, 1-1 Yanagido, Gifu 5011193, Japan.
}

Briefly, the animals were immobilized by intramuscular administration of zolazepam $\mathrm{HCl}$ and tiletamine $\mathrm{HCl}$ (Zoletil, Virbac, Carrors, France; $9 \mathrm{mg} / \mathrm{kg}$ of the estimated body weight). After immobilization, the penis was cleaned, and the bladder was then emptied and flushed with sterile physiological saline by catheterization (5-fr. polypropylene urinary catheter; Sovereign, Sherwood Medical, MO, U.S.A.). A collection catheter (8-fr. Sovereign) whose tip had been cut off to widen the hole was inserted into the urethra and held during collection to recover semen. A rectal probe with two copper electrodes connected to an electrical stimulator was then inserted 12 to $16 \mathrm{~cm}$ into the rectum. The animals were stimulated at increasing voltages of 1 to $7 \mathrm{~V}$ (7V was used for up to 14 of 20 stimuli). Each stimulus lasted $5 \mathrm{sec}$ followed by a $10 \mathrm{sec}$ pause. This cycle was repeated either until semen was ejaculated or 20 stimuli were given. When signs of a possible imminent ejaculation at the next stimuli were noted, an additional five stimuli were given. When semen was not ejaculated during the first set of 20 stimuli, another set was administered after a few min. When not enough semen was collected, another set of stimuli was administered. The maximum repetition of sets was three. Semen recovered in the collection catheter was forced into a test tube by gently applying air.

The semen was examined for characteristics immediately after collection except for examinations of intact acrosomes and abnormal morphology, which were carried out after fixation and arrival at the laboratory. Semen examination was performed according to the method of Kojima et al. [9] with some modifications. Briefly, the appearance of the ejaculate was examined and urine contamination was judged based on its yellow color. Ejaculate volume was measured with the 
aid of a micropipette. Sperm concentration was estimated with a hemocytometer. Motility was evaluated by subjective observation under a microscope $(\times 400)$ at $38^{\circ} \mathrm{C}$, and the results were shown as the percentage of motile (including non-progressive) spermatozoa (\% motility). Viability was assessed by a supravital stain with eosin and nigrosin. Spermatozoa were fixed by mixing with an equal volume of $2 \% \mathrm{v} / \mathrm{v}$ glutaraldeyde in $0.165 \mathrm{M}$ sodium cacodylate $/ \mathrm{HCl}$, $\mathrm{pH}$ 7.3. Slides were prepared with the glutaraldehyde-fixed sample, and a total of 100 spermatozoa were examined under a phase-contrast microscope $(\times 1,000)$ to determine the proportion $(\%)$ of morphologically abnormal spermatozoa (\% abnormal morphology, excluding abnormal acrosomes and cytoplasmic droplets). The proportion (\%) of spermatozoa with intact acrosomes was determined according to a method described elsewhere [16].

Semen cryopreservation was performed according to the method of our previous study $[11,12]$ with some modifications. Briefly, the diluent used was egg yolk-TRIS-citrateglucose extender [15] supplemented with $0.52 \mathrm{mg} / \mathrm{ml}$ of potassium penicillin $\mathrm{G}$ and $0.8 \mathrm{mg} / \mathrm{m} l$ of streptomycin sulphate (first extender). If the concentration of spermatozoa collected was over $200 \times 10^{6} / \mathrm{ml}$, the semen was diluted at room temperature with the diluent (first extender) to yield $100 \times 10^{6} / \mathrm{m} l$. If it was under $200 \times 10^{6} / \mathrm{m} l$, the semen was diluted 2-fold with diluent. The diluted semen was separated into 6 aliquots and then cooled down to $4^{\circ} \mathrm{C}$ in a refrigerator for $3 \mathrm{hr}$. Equal volumes of the second extender, consisting of the first extender supplemented with $8,12,14$, 16,20 , and $24 \%(\mathrm{v} / \mathrm{v})$ glycerol, respectively, were then added at $4{ }^{\circ} \mathrm{C}$. Thus, the final concentrations of glycerol were $4,6,7,8,10$, and $12 \%(\mathrm{v} / \mathrm{v})$. No specific time was set for glycerol equilibration. Six aliquots $(0.2 \mathrm{~m} l \mathrm{each})$ of the diluted semen were loaded at about $5^{\circ} \mathrm{C}$ into straws $(0.5 \mathrm{ml}$ capacity, original length $132 \mathrm{~mm}$; Fujihira Industry Co., Ltd., Tokyo) cut into $70 \mathrm{~mm}$ segments and sealed with straw powder. The reason for such a small volume of loaded semen was to make 6 aliquots of diluted semen from the limited volume of semen collected. The straws were cut short to prevent them from floating in the liquid nitrogen since loading the uncut straws with a small volume of diluted semen created an air pocket. The straws were placed in a styrofoam container (inner size: $17.5 \times 24.5 \times 17.5 \mathrm{~cm}$ ) $6 \mathrm{~cm}$ horizontally above the surface of the liquid nitrogen at a height of $8 \mathrm{~cm}$. The straws were kept thus for $15 \mathrm{~min}$, then immediately plunged into the liquid nitrogen and stored until examination. Straws were allowed to thaw in water at $38^{\circ} \mathrm{C}$ for a few minutes and were used immediately for examination. The frozen-thawed semen was examined in a way similar to the method for fresh semen, except that no examinations of appearance, ejaculate volume, or sperm concentration were conducted.

Data are presented as means $\pm \mathrm{SD}$. The percentage data were subjected to arc sine square root transformation before statistical analysis. Differences among the characteristics of frozen-thawed sperm in relation to glycerol concentrations of the diluent and of fresh semen in relation to appearance of ejaculates were tested by one-factor ANOVA. The differences found in ANOVA were tested with Fisher's protected least significant difference (PLSD) post-hoc test. Differences of $P<0.05$ were regarded as statistically significant.

One semen sample was collected from each animal. In examining fresh semen $(\mathrm{n}=7)$, the ejaculate volume $(\mathrm{m} l)$, sperm concentration $\left(\times 10^{6} / \mathrm{m} l\right)$, percent motility, percent viability, percent intact acrosomes, and percent abnormal morphology were found to be $0.59 \pm 0.45,221.4 \pm 130.9$, $77.1 \pm 11.1 \%, 93.9 \pm 2.3 \%, 95.4 \pm 2.2 \%$ and $49.7 \pm 32.9 \%$, respectively (Table 1). The main abnormal morphologies observed were simply coiled $(10.4 \pm 16.9 \%)$ and bent tails $(18.0 \pm 23.2 \%)$. The ejaculates were white transparent $(n=4)$ and white yellow transparent $(n=3)$. There were no significant differences in appearance, (white transparent and white yellow transparent) percent motility, viability, intact acrosomes, and abnormal morphology of fresh spermatozoa (Table 1).

The characteristics of frozen-thawed spermatozoa are shown in Table 2. There were no significant differences among the various glycerol concentrations in either percent motility or percent abnormal morphology of frozen-thawed spermatozoa. Percent viability and percent intact acrosomes of spermatozoa cryopreserved with 4 and $6 \%$ glycerol were significantly higher than those with 10 and $12 \%$ glycerol.

We have customarily used an egg yolk-citrate-based diluent with $8 \%$ glycerol in our laboratory for freezing Japanese black bear semen $[11,12]$. However, we have found percent viability and intact acrosomes of frozen-thawed spermatozoa to be better when freezing semen at lower glycerol concentrations of 4 and $6 \%$. The effect of glycerol concentrations lower than $4 \%$ was unclear in this study. The toxic effect of a high glycerol concentration clearly emerged at over $10 \%$ glycerol in this study. The glycerol concentration we used previously appeared to be unsuitable for freezing Japanese black bear semen. Concentrations ranging from 2 to $10 \%$ have been used to cryopreserve mammalian

Table 1. Characteristics of fresh semen collected by electroejaculation from Japanese black bears

\begin{tabular}{lcccc}
\hline Appearance $^{\text {a) }}$ & \% Motility & \% Viability & \% Intact acrosome & \% Abnormal morphology \\
\hline White transparent $(\mathrm{n}=4)$ & $77.5 \pm 12.6$ & $93.3 \pm 2.8$ & $94.5 \pm 2.6$ & $55.5 \pm 37.4$ \\
White yellow transparent $(\mathrm{n}=3)$ & $76.7 \pm 11.5$ & $94.7 \pm 1.5$ & $96.7 \pm 0.6$ & $42.0 \pm 31.6$ \\
\hline Total & $77.1 \pm 11.1$ & $93.9 \pm 2.3$ & $95.4 \pm 2.2$ & $49.7 \pm 32.9$ \\
\hline
\end{tabular}

a) Appearance was used for judgment of urine contamination. A yellow color indicates contamination of semen with urine. The values shown are means $\pm \mathrm{SD}$. 
Table 2. Characteristics of frozen-thawed spermatozoa cryopreserved with different glycerol concentrations from Japanese black bears

\begin{tabular}{cllcc}
\hline Glycerol concentration (\%) & \% Motility & \% Viability & \% Intact acrosome & \% Abnormal morphology \\
\hline 4 & $23.6 \pm 13.5$ & $63.3 \pm 19.6^{\mathrm{a})}$ & $53.9 \pm 12.5^{\mathrm{a})}$ & $47.3 \pm 37.2$ \\
6 & $25.0 \pm 12.9$ & $60.1 \pm 26.1^{\mathrm{a})}$ & $53.9 \pm 14.2^{\mathrm{a})}$ & $45.1 \pm 35.9$ \\
7 & $20.7 \pm 6.7$ & $57.6 \pm 22.7^{\mathrm{a})}$ & $43.9 \pm 19.4^{\mathrm{a}, \mathrm{b})}$ & $45.9 \pm 31.7$ \\
8 & $25.7 \pm 12.4$ & $48.3 \pm 20.3^{\mathrm{a}, \mathrm{b})}$ & $39.0 \pm 12.7^{\mathrm{a}, \mathrm{b}, \mathrm{c})}$ & $51.6 \pm 34.9$ \\
10 & $15.0 \pm 7.1$ & $26.0 \pm 12.1^{\mathrm{b})}$ & $29.0 \pm 14.6^{\mathrm{b}, \mathrm{c}}$ & $50.9 \pm 35.8$ \\
12 & $15.7 \pm 6.1$ & $30.6 \pm 23.5^{\mathrm{b})}$ & $24.3 \pm 22.6^{\mathrm{c})}$ & $48.4 \pm 33.1$ \\
\hline
\end{tabular}

The values shown are means \pm SD. Groups with different superscripts in the same column are significantly differnt $(P<0.05)$.

sperm [1]. Concentrations of $4.7 \%$ and $4-5 \%$ have been used for freezing the semen of closely related species, such as the brown bear [7] and giant panda [13, 18], respectively. Our results corresponded to the conventional glycerol concentrations used in other ursids.

Optimal cryoprotectant concentrations are dependent on cooling rates as well as species [4, 19]. Our cryopreservation method required no special equipment and proved useful for non-domestic and non-laboratory animals. In our method, straws were cooled by liquid nitrogen vapor in a styrofoam container. Generally, lower glycerol concentrations require faster cooling rates [19]. Using this method, a glycerol concentration of 4 to $6 \%$ may be optimal for freezing Japanese black bear semen with in the range tested.

The quality of spermatozoa was greatly reduced after freezing and thawing in this study. The current results for frozen-thawed spermatozoa were inferior to our previous results (\% motility $36.3 \pm 17.7 \%$, \% viability $63.2 \pm 10.4 \%$, $\%$ intact acrosomes $73.6 \pm 10.6 \%$ and $\%$ abnormal morphology $26.6 \pm 15.4 \%$; cryopreserved with $8 \%$ glycerol) [12] The reason for this may be that the characteristics of the fresh semen used were poor originally. In this study, secondary sperm abnormalities, i. e., simply coiled or bent tails, were observed frequently in fresh sperm. Because there were no significant differences in the appearance of the fresh sperm, high abnormal morphology rates were not considered to be caused by urine contamination primarily. The reason for many of the abnormalities and the poor freezing tolerance in this study were unclear.

In some species, including rams [4], stallions [3], boars [2], and Rhesus monkeys (Macaca mulatta) [17], different glycerol concentrations affect frozen-thawed sperm motility. However, neither sperm motility nor abnormal morphology was affected by the various glycerol concentrations used in this Japanese black bears study. The plasma membrane and acrosome are particularly vulnerable to the stresses of freezing and thawing [19]. Sperm viability and intact acrosomes would be a sensitive indicator of glycerol toxicity.

In conclusion, sperm frozen with 4 and $6 \%$ glycerol exhibited a higher percent of viability and intact acrosomes after thawing than that with higher concentrations, leading us to conclude that the most suitable glycerol concentration for freezing Japanese black bear semen would be $4-6 \%$ within the range tested.

The authors wish to thank Dr. R. Nakashita of the United Graduate School of Agricultural Science, Tokyo University of Agriculture and Technology, as well as the members of the Student Chapter of the Japanese Society of Zoo and Wildlife Medicine for their valuable assistance in semen collection. This work could not have been conducted without the kind cooperation of Mr. A. Izumi, Mr. M. Suzuki, and Mr. S. Kikuchi at the Ani Mataginosato Bear Park, Akita, Japan. This study was supported in part by a Grantin-Aid for Scientific Research (The 21st Century Center-ofExcellence Program, E-1 and 15380215) from the Ministry of Education, Culture, Sports, Science and Technology of Japan.

\section{REFERENCES}

1. Agca, Y. and Critser, J. K. 2002. Semin. Reprod. Med. 20: 1523.

2. Buhr, M. M., Fiser, P., Bailey, J. L. and Curtis, E. F. 2001. J. Androl. 22: 961-969.

3. Cochran, J. D., Amann, R. P., Froman, D. P. and Pickett, B. W. 1984. Theriogenology 22: 25-38.

4. Fiser, P. S. and Fairfull, R. W. 1984. Cryobiology 21: 542-551.

5. Hazumi, T. 1999. pp. 207-211. In: Bears Status Survey and Conservation Action Plan (Servheen, C., Herrero, S. and Peyton, B. eds.), IUCN, Gland, Switzerland and Cambrige, U. K.

6. Holt, W. V. 2000. Theriogenology 53: 47-58.

7. Ishikawa, A., Matsui, M., Sakamoto, H., Katagiri, S. and Takahashi, Y. 2002. J. Vet. Med. Sci. 64: 373-376.

8. Ishikawa, A., Matsui, M., Tsuruga, H., Sakamoto, H., Takahashi, Y. and Kanagawa, H. 1998. J. Vet. Med. Sci. 60: 965968.

9. Kojima, E., Tsuruga, H., Komatsu, T., Murase, T., Tsubota, T. and Kita, I. 2001. Theriogenology 55: 717-731.

10. McLaughin, E. A., Ford, W. C. L. and Hull, M. G. R. 1992. J. Reprod. Fertil. 95: 749-754.

11. Okano, T., Murase, T. and Tsubota, T. 2004. J. Vet. Med. Sci. 66: $1371-1376$.

12. Okano, T., Murase, T., Yayota, C., Komatsu, T., Miyazawa, K., Asano, M. and Tsubota, T. Anim. Reprod. Sci. (in press).

13. Olson, M. A., Yan, H., DeSheng, L., Hemin, Z. and Durrant, B., 2003. Zoo. Biol. 22: 335-345.

14. Platz, C. C. Jr., Wildt, D. E., Howard, J. G. and Bush, M. 1983. J. Reprod. Fertil. 67: 9-12.

15. Rota, A., Strom, B., Linde-Forsberg, C. and Rodriguez-Martinez, H. 1997. Theriogenology 47: 1093-1101. 
16. Shams-Borhan, G. and Harrison, R. A. 1981. Gamete. Res. 4: 407-432.

17. Si, W., Zheng, P., Li, Y., Dinnyes, A. and Ji, W. 2004. Am. J. Primatol. 62: 301-306.

18. Spindler, R. E., Huang, Y., Howard, J. G., Wang, P., Zhang,
H., Zhang, G. and Wildt, D. E. 2004. Reproduction 127: 547556.

19. Watson, P. F. 1990. pp. 747-869. In: Marshall's Physiology of Reproduction, 4th ed., vol. II. Reproduction in the Male (Lamming, G. E. ed.), London, Churchill Livingstone. 